\title{
Study on multimodal functional magnetic resonance images of schizophrenic patients with initial negative symptom.
}

\author{
Ruiyu Huang ${ }^{1}$, Hui'an $\mathrm{Li}^{2}$, Lizhe Sun ${ }^{3 *}$, Baogang $\mathrm{Xu}^{1}$, Xingzhi Ma1 \\ ${ }^{1}$ MRI Room, Second Affiliated Hospital of Shaanxi University of Chinese Medicine, Xianyang, Shaanxi, PR China \\ ${ }^{2}$ Department of Radiology, People's Hospital of Ankang City, Ankang, Shaanxi, PR China \\ ${ }^{3}$ Second Affiliated Hospital of Shaanxi University of Chinese Medicine, Xianyang, Shaanxi, PR China
}

\begin{abstract}
Objective: To study the characteristics of the multimodal functional Magnetic Resonance Imaging (fMRI) of schizophrenia patients with initial negative symptom.

Methods: From January 2014 to January 2016, 30 cases of schizophrenia patients with the initial negative symptom and 30 cases of schizophrenia patients with the initial positive symptom were involved in our study. Besides, other 30 cases were matched into the healthy control based on the gender, age and education level. The 3D structure Magnetic Resonance Imaging (MRI) data and functional MRI data of all patients were collected. The voxel-based morphometry was used to analyse different brain regions in structure MRI data among those three groups. In addition, we analysed the correlation between all functions of the whole brain in the resting state and different brain regions.

Results: The volume of the gray matter in the right dorsolateral prefrontal lobe of the patients with initial negative symptom was significantly higher than that of patients with initial positive symptom. Moreover, in the control group, the whole brain's functional connectivity was decreased in the dorsolateral prefrontal cortex under a resting state. The difference was mainly on functional connectivity between the right dorsolateral prefrontal cortex-right medial prefrontal cortex and right dorsolateral prefrontal cortex-right caudate nucleus. The $Z$ value of right medial prefrontal cortex in the negative symptom group was significantly lower than those of other two groups. The right caudate nucleus $Z$ value was significantly higher than those of other two groups. When the $\mathrm{N}$-back working memory task was given, the different brain regions in activations among the three groups were the left dorsolateral prefrontal cortex, medial prefrontal cortex/anterior cingulated cortex and posterior cingulated cortex, the inhibition degree against the medial prefrontal cortex/anterior cingulated cortex and posterior cingulated cortex of patients with the initial negative symptom as primary type was significantly decreased and the dorsolateral prefrontal activation was significantly increased.

Conclusion: It has a significant value to diagnose and identify the schizophrenia with the initial negative symptom through the characteristics of the multimodal fMRI.
\end{abstract}

Keywords: Initial negative symptom, Schizophrenia, Multimodal fMRI.

Accepted on October 23, 2017

\section{Introduction}

The prevalence of schizophrenia is about $1 \%$, the incidence peak is in early adulthood, and its clinical characteristics mainly include perception disorder, thinking disorder, affective disorder, behavior disorder, neurocognitive disorder, and so on [1]. The schizophrenia factor analysis method believes that the three-dimensional symptom grouping can be more conducive to understanding the disease and researching the pathomechanism [2]. The three dimensions mean positive symptom (hallucination, delusion), negative symptom (poverty of speech, decrease in the spontaneous movement, apathy) and depersonalization symptom (formal thought disorder, bizarre behavior, inappropriate emotion) [3]. Meta's analysis [4] found that the negative symptom might be correlated with the dorsolateral and anterolateral prefrontal cortex and ventral striatum, the positive symptom might be correlated with the medial prefrontal cortex and medial temporal lobe and the depersonalization symptom might be correlated with the dorsolateral prefrontal lobe. Our study investigated the neurobiology mechanism of negative and positive symptoms of schizophrenia from different aspects through the multimodal fMRI analysis method. This will provide references to individualized treatment of the patients with different clinical manifestations. 


\section{Materials and Methods}

\section{General information}

From January 2014 to January 2016, patients with initial schizophrenia diagnosed in Second Affiliated Hospital of Shaanxi University of Chinese Medicine were contiguously selected, including 30 cases with predominantly negative symptoms and 30 cases with predominantly positive symptoms (Table 1).

Inclusion criteria: 1 . In line with the diagnosis standard for the first episode of schizophrenia in American Diagnostic and Statistical Manual of Mental Disorders (DSM-IV); 2. Age from 18 to 45 y old; 3 . The disease duration $\leq 18$ months; 4 . Cultural education time $\geq 9 \mathrm{y}$.

Exclusion criteria: 1. Brain diseases, such as cancer, epilepsy post stroke; 2. Severe anxiety or depression, mental stimulation, alcohol, recreational drug and drug abuse or dependence; 3. Administration of antipsychotic drugs; 4. Underlying diseases, such as severe organ dysfunctions such as heart dysfunction, liver dysfunction, lung dysfunction, kidney dysfunction, brain dysfunction, and taboo in MRI examination; 5. Pregnant and lactating women received electric shock treatment; 6 . The image data was incomplete or could not be analysed. The study has been approved by the ethics committee of our hospital and the informed consents of patients and their families have been obtained, 30 cases were selected and matched into the healthy control group based on the gender, age and education level in the same period. The baseline data have comparability among the three groups.

Table 1. General information of all patients.

\begin{tabular}{|c|c|c|c|c|c|}
\hline Group & $\mathbf{n}$ & $M / F$ & Age & $\begin{array}{l}\text { Disease } \\
\text { duration } \\
\text { (months) }\end{array}$ & $\begin{array}{l}\text { Education } \\
\text { time } \\
\text { (years) }\end{array}$ \\
\hline $\begin{array}{l}\text { Negative } \\
\text { group }\end{array}$ & 30 & $14 / 16$ & $32.5 \pm 8.2$ & $5.3 \pm 2.4$ & $15.2 \pm 3.7$ \\
\hline Positive symptom group & 30 & $15 / 15$ & $33.4 \pm 9.3$ & $5.4 \pm 2.3$ & $14.6 \pm 3.5$ \\
\hline Control group & 30 & $16 / 14$ & $35.6 \pm 8.6$ & - & $15.3 \pm 3.9$ \\
\hline$F / X^{2}$ & & 0.01 & 0.21 & 0.15 & 0.23 \\
\hline $\mathrm{P}$ & & 1.000 & 0.862 & 0.923 & 0.834 \\
\hline
\end{tabular}

\section{Research methods}

The 3D structure MRI data and the functional MRI data of subjects in the resting state and N-back task state were collected, the voxel-based morphometry was used to analyse different brain regions in structure MRI data among the three groups, and then the analysis was carried out on the connectivity of all functions of the whole brain in the resting state with different brain regions as the seed regions and on the activity difference in the task-related brain regions among the three groups in the N-back task state.

\section{D structure MRI data preprocessing and analysis}

MRI data analysis is mainly based on MATLAB 7.10 to process the brain structure image through SPM8The preprocessing on the $3 \mathrm{D}$ structure MR data through the VoxelBased Morphometry (VBM) mainly includes standardization, segmentation, adjustment, smoothing and other steps, to obtain the image after adjusting the gray matter and white matter, which represents the volume of the brain gray matter and white matter. With TIV as a covariate, the analysis of covariance was applied to carry out the model statistics and test the difference in the brain gray matter volumes among the three groups, the significant difference threshold was set as $p<0.001$. The Rest software was applied to extract the absolute values of the gray matters in the brain regions of the subjects with a significant difference in the brain gray matter volumes.

\section{The preprocessing and analysis of fMRI data in the resting and task states}

Considering the uniform field effect, the images at the former two time points were eliminated; The time of the images at the remaining 248 time points was corrected; the head movement correction parameters included the translation among $\mathrm{X}, \mathrm{Y}$ and $\mathrm{Z}$ directions and the rotation around these three directions, and the translation towards each direction was less than $3 \mathrm{~mm}$, and the rotation is less than $3^{\circ}$; fMRI images and SPM8 standard EPI templates were standardized, fMRI data were re-sampled with $3 \times 3 \times 3 \mathrm{~mm}^{3}$ size as voxel unit; the space was smoothed on the standardized image with $8 \times 8 \times 8 \mathrm{~mm}^{3}$ FWHM Gaussian kernel function; The linear regression method was used to exclude the constant term, linear drift, whole brain mean signal fluctuation and the influence of the head movement on the signal. The rational band-pass filtering processing was carried out on each voxel time, with the limited range of $0.01 \sim 0.08 \mathrm{~Hz}$. Resting-state data analysis: With significantly different brain regions in brain gray matter volume as the Region of Interest (ROI), the re-sampling was carried out based on $3 \times 3 \times 3 \mathrm{~mm}^{3}$ so that the ROI could be matched with fMRI data. The corresponding ROI mean time sequence was calculated with ROI as seed points and taken as the reference time sequence, the coefficients related to each voxel time sequence of the whole brain were calculated one by one and taken as a measure of the functional connectivity strength of ROI and each voxel, thereby the corresponding ROI functional connectivity network could be obtained. The first level analysis was the individual level analysis, through the analysis on each individual pixel by pixel, the activated brain regions were tested in 0-back and 2-back states; the second level analysis was the level analysis among groups, through one-way analysis of variance, the difference in the brain region activations among the positive symptom group, negative symptom group and healthy control group was tested.

\section{Statistical analysis}

SPSS20.0 software was used for statistical analysis, the measurement data was expressed in mean \pm standard deviation, the comparison among multiple groups was made through one- 
way ANOVA analysis, the pairwise comparison was tested through LSD-t test method and the comparison between the two groups was tested through independent sample t-test method. The count data was expressed in the number of cases (\%) and the comparison among the groups were tested through Chi-square test method; The correlation analysis was tested through Pearson or Spearman test method. $\mathrm{P}<0.05$ indicates that the difference is statistically significant

\section{Results}

\section{Analysis and comparison of brain gray matter volume $\boldsymbol{V B M}$}

The gray matter volume in the Dorsolateral Prefrontal Cortex (DLPFC) in the negative symptom group was significantly higher than that in the positive symptom and control groups, and the lowest value was in the control group, the difference was statistically significant $(\mathrm{P}<0.05)$ (Figure 1).
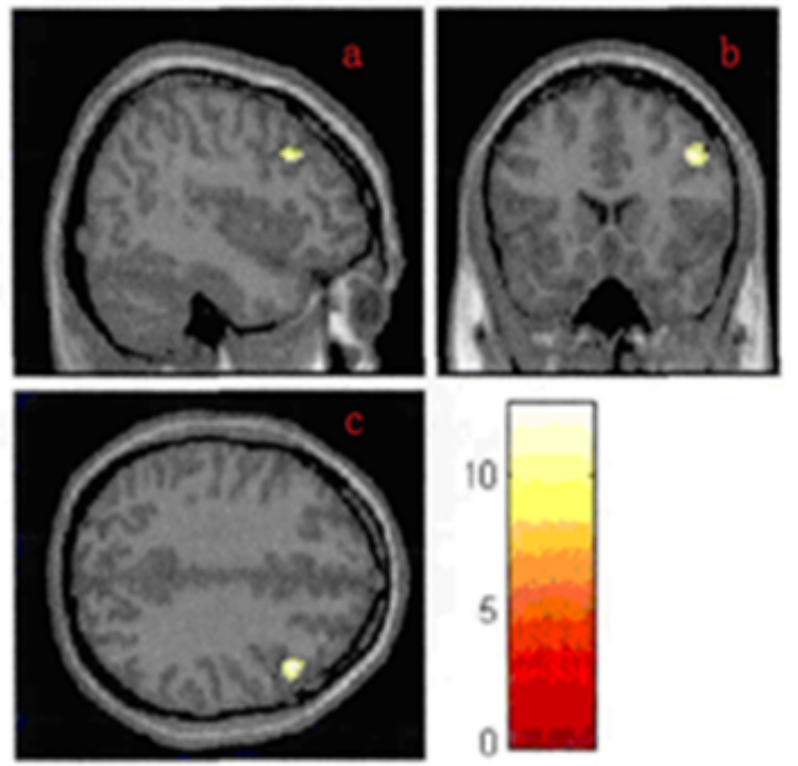

Figure 1. Comparison of brain gray matter volume.

\section{Resting-state functional connectivity analysis}

The connectivity of all functions of the whole brain in the right dorsolateral prefrontal lobe in resting state was significantly decreased and the difference was mainly focused on the functional connectivity among the right dorsolateral prefrontal lobe-the right medial prefrontal lobe and the right dorsolateral prefrontal lobe-the right caudate nucleus. The right medial prefrontal cortex $\mathrm{Z}$ value in the negative symptom group was significantly lower than other two groups. The right caudate nucleus $\mathrm{Z}$ value was significantly higher than that in other two groups. No difference was found between positive symptom group and control group (Figure 2 and Table 2).
A

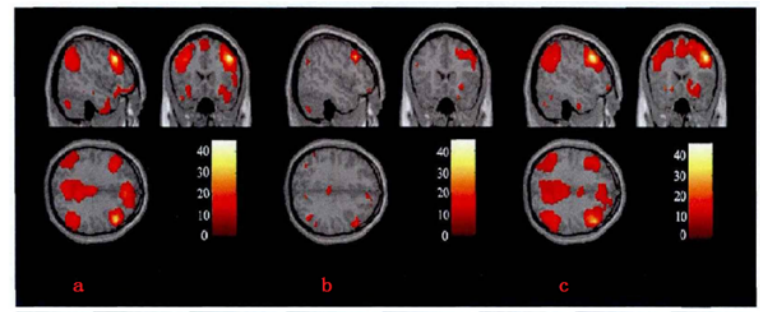

B

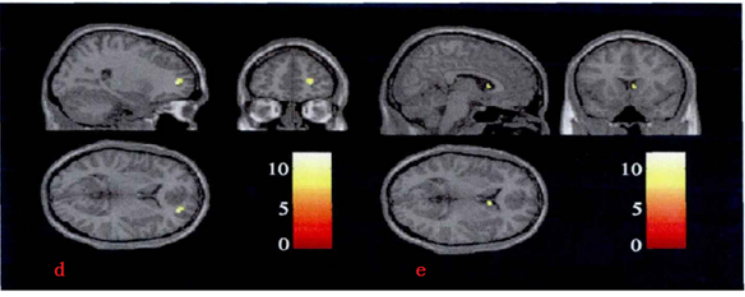

Figure 2. Analysis of functional connectivity in the resting state. A. The $Z$ value distribution figure for the functions of the whole brain in the right dorsolateral prefrontal lobe (a: negative symptom group; $b$ : positive symptom group; $c$ : control group); $B$. The significantly different brain regions in the functional connectivity of the right dorsolateral prefrontal lobe (d: right medial prefrontal cortex; e: right caudate nucleus).

$\mathbf{A}$

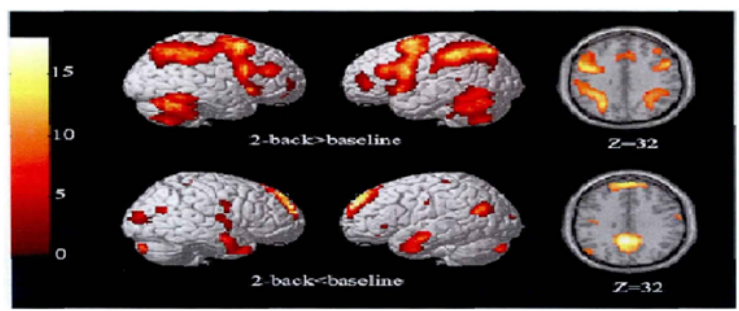

B

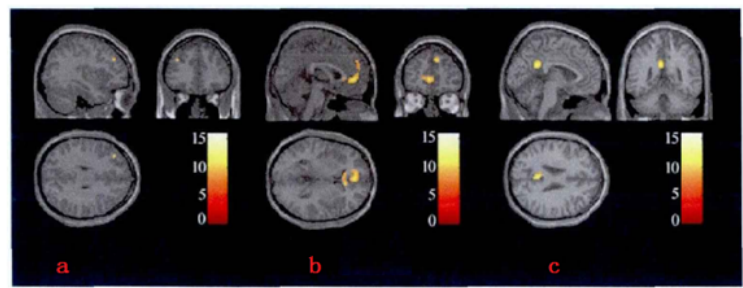

Figure 3. Task state functional magnetic resonance analysis. A. The distribution of the activated and inhibited brain regions of the subjects when they completed 2-back task; B. The significantly different brain regions in brain activity (a: left dorsolateral prefrontal lobe; $b$. medial prefrontal cortex/anterior cingulated cortex; $c$. posterior cingulated cortex).

\section{Task state functional magnetic resonance analysis}

When N-back working memory task was given, the different brain regions inactivation among all three groups were the left dorsolateral prefrontal cortex, medial prefrontal cortex/anterior cingulated cortex and posterior cingulated cortex, the inhibition degree against the medial prefrontal lobe/ anterior cingulated cortex and posterior cingulated cortex in the negative symptom group was significantly decreased and the dorsolateral prefrontal activation was significantly increased. There was no 
difference between the positive symptom group and the control group by comparison (Figure 3 and Table 3).

Table 2. Resting-state functional connectivity analysis ( $Z$ value).

\begin{tabular}{llllll}
\hline Brain area & Negative symptom group & Positive symptom group & Control group & F & \\
\hline $\begin{array}{l}\text { Right medial prefrontal } \\
\text { cortex }\end{array}$ & $0.15 \pm 0.05$ & $0.36 \pm 0.10$ & $0.41 \pm 0.12$ & 0.015 \\
\hline \begin{tabular}{l} 
Right caudate nucleus \\
\hline
\end{tabular} & $0.38 \pm 0.12$ & $0.18 \pm 0.06$ & $0.17 \pm 0.03$ & 5.12 \\
\hline
\end{tabular}

Table 3. Task state functional magnetic resonance analysis (BOLD signal).

\begin{tabular}{|c|c|c|c|c|c|c|c|}
\hline Brain area & $\begin{array}{l}\text { Negative } \\
\text { group }\end{array}$ & symptom & $\begin{array}{l}\text { Positive } \\
\text { group }\end{array}$ & symptom & Control group & $\mathbf{F}$ & $\mathbf{P}$ \\
\hline Left dorsolateral prefrontal lobe & $1.23 \pm 0.25$ & & $0.42 \pm 0.06$ & & $0.32 \pm 0.05$ & 6.33 & 0.029 \\
\hline Medial prefrontal cortex/anterior cingulated cortex & $-0.12 \pm 0.06$ & & $-0.63 \pm 0.23$ & & $-0.72 \pm 0.21$ & 6.2 & 0.017 \\
\hline Posterior cingulated cortex & $-0.56 \pm 0.11$ & & $-0.86 \pm 0.21$ & & $-0.92 \pm 0.23$ & 5.86 & 0.035 \\
\hline
\end{tabular}

\section{Discussion}

It is mainly manifested as social withdrawal, lack of motivation, anhedonia and apathy, its pathological mechanism is correlated with the prefrontal cortex and subcortical structure disorders, among which, the dorsolateral prefrontal lobe dysfunction is correlated with abnormal connectivity of other brain regions (such as parietal cortex, thalamus and striatum) and executive dysfunction of schizophrenia, which may be the common neurobiological mechanism for schizophrenia negative symptom and cognitive disorder $[5,6]$.

The structure neuroimaging study [7] found that the volume of the working memory-related brain regions (such as the frontal and temporal lobes) of the schizophrenia was decreased. DLPFC was an important working memory related subcortical region, the decrease degree of DLPFC gray matter volume was correlated with the cognitive dysfunction and the severity of the negative symptoms [8]. The working memory deficit was correlated with the severity of the negative symptoms of schizophrenia and might affect the social function of the patients [9]. When performing the working memory tasks by the Schizophrenia patients, they might have longer reaction time, low correction rate and worse and worse working memory performance as the increase in the working memory load. DLPFC was activated and enhanced in patients with schizophrenia and positively correlated with the working memory performances [10]. At low memory load, DLPFC could reach the activation peak and the activation would be decreased with the increase in the memory load.

Our study results found that the volume of the gray matter in the right dorsolateral prefrontal lobe of the patients with the initial negative symptom as primary type was significantly higher than that of patients with the initial positive symptom as primary type and in the control group. The magnetic functional spectroscopy studies found that [11] the decrease in the concentration of $\mathrm{N}$-acetyl aspartate in DLPFC suggested the neuronal neurotransmitter metabolism and it was correlated with the antipsychotic drug treatment. If the DLPFC gray matter volume was increased, considering the disease duration of schizophrenia patients with initial symptoms was shorter and no medication had been administrated, the function compensation possibility might be increased. The whole brain's functional connectivity was decreased in the dorsolateral prefrontal cortex under a resting state. The difference was mainly focusing on functional connectivity between the right dorsolateral prefrontal cortex-right medial prefrontal cortex and right dorsolateral prefrontal cortex-right caudate nucleus. The right medial prefrontal cortex $\mathrm{Z}$ value in the negative symptom group was significantly lower than other two groups. The right caudate nucleus $Z$ value was significantly higher than other two groups. No difference was found between positive symptom group and control group. DLPFC-caudate nucleus constituted the cognitive loop; the patients with negative symptoms had poor cognitive functions and should mobilize more additional cognitive resources while performing cognitive tasks, the enhancement in the connectivity of DLPFC-caudate nucleus was in line with the clinical practice [12]. When the N-back working memory task was given, the different brain regions in activations among the three groups were the left dorsolateral prefrontal cortex, Medial Prefrontal Cortex/Anterior Cingulated Cortex (MPFC/ ACC) and Posterior Cingulated Cortex (PCC), the inhibition degree against the medial prefrontal cortex/ anterior cingulated cortex and posterior cingulated cortex of patients with the initial negative symptom as primary type was significantly decreased and the dorsolateral prefrontal activation was significantly increased. MPFC/ACC and PCC were cortical midline structures, playing important roles during the selfcognition and social cognition information processing [13]. In MPFC/ACC and PCC brain region, the inhibition activity was decreased so that the cortical midline structure was in the state of disinhibition and the reality test power was decreased, 
leading to the attention and motivation resource misallocation for the patients at the time of perception and adaptation to the external environment [14]. DLPFC and MPFC were important network nodes positively and negatively related to the tasks, the mutual coordination and competition between the two networks were the neurobiological basis for good cognitive performance [15].

In conclusion, it has a significant value to diagnose and identify the schizophrenia with the initial negative symptom as primary type through the characteristics of the multimodal fMRI.

\section{References}

1. Kimura H, Kanahara N, Sasaki T, Komatsu N, Ishige M, Muneoka K, Ino H, Yoshimura K, Yamanaka H, Suzuki T, Komatsu H, Watanabe H, Shimizu E, Iyo M. Risperidone long-acting injectable in the treatment of treatment-resistant schizophrenia with dopamine supersensitivity psychosis: results of a 2-year prospective study, including an additional 1-year follow-up. J Psychopharmacol 2016; 7: $1-2$.

2. Barch DM, Marder SR, Harms MP, Jarskog LF, Buchanan RW, Cronenwett W, Chen LS, Weiss M, Maguire RP, Pezous N, Feuerbach D, Lopez-Lopez C, Behrje RB, Gomez-Mancilla B. Task-related fMRI responses to a nicotinic acetylcholine receptor partial agonist inschizophrenia: a randomized trial. Prog Neuropsychopharmacol Biol Psychiatry 2016; 6: 15-16.

3. Kosger F, Essizoglu A, Sonmez I, Gulec G, Genek M, Akarsu O. The relationship between violence and clinical features, insight and cognitive functions in patients with schizophrenia. Turk Psikiyatri Derg 2016; 27: 1-2.

4. Palaniyappan L, Maayan N, Bergman H, Davenport C, Adams CE, Soares-Weiser K. Voxel-based morphometry for separation of schizophrenia from other types of psychosis in first episode psychosis. Cochrane Database Syst Rev 2015; 8: 011021.

5. Czepielewski LS, Wang L, Gama CS, Barch DM. The relationship of intellectual functioning and cognitive performance to brain structure in schizophrenia. Schizophr Bull 2016; 7: 14-15.

6. Jung SI, Woo J, Kim YT, Kwak SG. Validation of the Korean-version of the clinical assessment interview for negative symptoms of schizophrenia (CAINS). J Korean Med Sci 2016; 31: 1114-1120.

7. Kar SK, Jain M. Current understandings about cognition and the neurobiological correlates in schizophrenia. J Neurosci Rural Pract 2016; 7: 412-418.
8. Dutta M, Nath K, Baruah A, Naskar S. A clinical study of neurological soft signs in patients with schizophrenia. J Neurosci Rural Pract 2016; 7: 393-399.

9. Mulligan LD, Haddock G, Emsley R, Neil ST, Kyle SD. High resolution examination of the role of sleep disturbance in predicting functioning and psychotic symptoms in schizophrenia: a novel experience sampling study. J Abnorm Psychol 2016; 6: 17-18.

10. Sullivan PF. Schizophrenia: Hope on the Horizon. Cerebrum 2015; 2015.

11. Sarpal DK, Lencz T, Malhotra AK. In support of neuroimaging biomarkers of treatment response in firstepisode schizophrenia. Am J Psychiatry 2016; 173: 732-733.

12. Torres US, Duran FL, Schaufelberger MS, Crippa JA, Louza MR, Sallet PC, Kanegusuku CY, Elkis H, Gattaz WF, Bassitt DP, Zuardi AW, Hallak JE, Leite CC, Castro CC, Santos AC, Murray RM, Busatto GF. Patterns of regional gray matter loss at different stages of schizophrenia: a multisite, cross-sectional VBM study in first-episode and chronic illness. Neuroimage Clin 2016; 12: 1-15.

13. Tikasz A, Potvin S, Lungu O, Joyal CC, Hodgins S, Mendrek A, Dumais A. Anterior cingulate hyperactivations during negative emotion processing among men with schizophrenia and a history of violent behavior. Neuropsychiatr Dis Treat 2016; 12: 1397-1410.

14. Liu X, Zhuo C, Qin W, Zhu J, Xu L, Xu Y, Yu C. Selective functional connectivity abnormality of the transition zone of the inferior parietal lobule in schizophrenia. Neuroimage Clin 2016; 11: 789-795.

15. Goghari VM, Harrow M. Twenty-year multi-follow-up of different types of hallucinations in schizophrenia, schizoaffective disorder, bipolar disorder, and depression. Schizophr Res 2016; 6: 14-15.

\section{*Correspondence to}

Lizhe Sun

Second Affiliated Hospital of Shaanxi University of Chinese Medicine

Shaanxi

PR China 